\title{
INFLUÊNCIA DA INCORPORAÇÃO DO EXTRATO DE BARBATIMÃO EM FILMES DE AMIDO E CASEÍNA
}

\author{
Allan Remor Lopes ${ }^{1}$, Douglas Cardoso Dragunski², Leandro Ferreira Bonfim Júnior ${ }^{3}$, Camila Botin
} Francisco ${ }^{4}$, Josiane Caetano ${ }^{2}$

\begin{abstract}
RESUMO - O objetivo desse trabalho foi investigar os efeitos do extrato de barbatimão nas propriedades físicas e estruturais de filmes de amido e caseína. Foram analisados filmes de amido (A), amido e caseína (AC), amido com barbatimão $(\mathrm{AB})$, e amido e caseína com barbatimão $(\mathrm{ACB})$. Os resultados indicaram que a espessura não se alterou, os filmes AB e ACB são menos solúveis, com 20,41 e 20,5\% de solubilidade e mais opacos que os filmes sem extrato de barbatimão, com 5,57 e 6,64 Abs600. $\mathrm{mm}^{-1}$, respectivamente. Com a presença do barbatimão, os filmes se tornaram mais escuros e amarelados. A análise morfológica pela microscopia eletrônica de varredura (MEV) indicou que os filmes com o extrato de barbatimão se tornaram mais ásperos e heterogêneos. Este estudo demonstrou que o extrato de barbatimão pode ser incorporado em filmes tanto de amido, quanto de amido e caseína.
\end{abstract}

Palavras-chave: Biopolímero, filmes biodegradáveis, Stryphnodendron adstringens.

\section{INFLUENCE OF BARBATIMÃO EXTRACT INCORPORATION IN STARCH AND CASEIN FILMS}

\begin{abstract}
The objective of this study was to investigate the effects of the barbatimão extract on the physical and structural properties of starch and casein films. Films of starch (A), starch and casein (AC), starch with barbatimão $(A B)$, and starch and casein with barbatimão were analyzed. The results indicated that the thickness did not change, AB and ACB films are less soluble, with 20,41 and 20,5\% and more opaque than films without barbatimão extract, with 5,57 and 6,64, Abs600. $\mathrm{mm}^{-1}$, respectively. The barbatimão presence, the films became more darker and yellowish. The morphological analysis by scanning electron microscopy (SEM) indicated the films with the barbatimão extract became rougher and heterogeneous. This study demonstrated that barbatimão extract can be incorporated into films of both starch, starch and casein.
\end{abstract}

Keywords: Biopolymer, biodegradable films, Stryphnodendron adstringens.

\footnotetext{
${ }^{1}$ Discente Programa de Pós-Graduação em Engenharia Agrícola (PGEAGRI) - Universidade Estadual do Oeste do Paraná (UNIOESTE). allanremorlopes@gmail.com*Autor para correspondência.

${ }^{2}$ Docente Centro de Engenharias e Ciências Exatas - Universidade Estadual do Oeste do Paraná (UNIOESTE).

${ }^{3}$ Discente Universidade Paranaense (UNIPAR).

${ }^{4}$ Discente Programa de Pós-Graduação em Química (PQU) - Universidade Estadual de Maringá (UEM).
} 


\section{INTRODUÇÃO}

O uso excessivo de materiais derivados do petróleo vem acumulando uma grande quantidade de resíduos, pois os mesmos possuem uma baixa capacidade de degradar-se, e são classificados como agentes altamente poluentes. Diversas alternativas foram criadas para a redução do problema: A reutilização de embalagens, a reciclagem e outras formas de recuperação de resíduos. Contudo, estas medidas não obtiveram uma redução significativa desses resíduos (Rodríguez et al., 2012).

Devido aos problemas ocasionados pela disposição dos polímeros sintéticos, motivou o desenvolvimento, produção e aplicação de polímeros biodegradáveis. Como alternativa aos polímeros sintéticos há um grande interesse no uso de embalagens biodegradáveis feitos de fontes renováveis, como o amido e a caseína (Fernandes et al., 2015). Estes materiais possuem as vantagens de serem abundantes, renováveis, terem baixo custo e biodegradáveis (Lafargue, Lourdin \& Doublier, 2007).

A utilização de filmes biodegradáveis pode ajudar a resolver o problema, através da substituição parcial dos materiais não degradáveis (Martins, Cerqueira \& Vicente, 2012). Os filmes biodegradáveis são finas camadas biodegradáveis que podem ser aplicadas sobre a superfície dos alimentos, e fornecem uma barreira de gases, aumentando sua validade e qualidade (Vermeiren et al., 1999).

Dentre os agentes formadores de filmes, a caseína produz filmes flexíveis, transparentes e sem odores. Para Correa-Betanzo et al. (2011), a caseína possui uma estabilidade térmica e pode formar filmes a partir de soluções aquosas. Os filmes à base de amido apresentam características físicas apropriadas, uma vez que são isotrópicos, inodoros, não tóxicos, biodegradáveis, sem sabor, incolores e constituem uma boa barreira para a transferência de oxigênio (Yan et al., 2012).

Uma maneira viável de melhorar a funcionalidade dos filmes é através da incorporação de compostos ativos (antioxidantes e agentes antimicrobianos), que podem controlar a deterioração e a proliferação de patógenos nos alimentos durante o transporte e armazenamento (Moure et al., 2001).

O barbatimão (Stryphnodendron adstringens), pertencente a família Fabaceae, é uma espécie brasileira distribuída pelo cerrado brasileiro (Castro et al., 2009).
Há muito tempo o barbatimão já é utilizado no Brasil, como agente antiséptico e antiprotozoário, e já demonstrou eficácia contra Trypanosoma e Leishmania, além de ser usado na cicatrização de feridas (Nascimento et al., 2013; Oliveira et al., 2014). O interesse na utilização do extrato de barbatimão está relacionado aos seus compostos mencionados como um forte antioxidante e agente antimicrobiano.

Neste contexto, este trabalho teve o objetivo avaliar os efeitos do extrato de barbatimão nas propriedades físicas e estruturais de filmes de amido e caseína.

\section{MATERIAL E MÉTODOS}

A casca do barbatimão foi adquirida no comércio local de Umuarama, Paraná. O extrato foi obtido pela técnica denominada de extração hidroalcoólica, no qual foi adicionado $0,3 \mathrm{~L}$ de etanol em $400 \mathrm{~g}$ de barbatimão e posteriormente utilizando um rotoevaporador (Tecnal TE-210) por um período de 3 horas para a total evaporação do solvente. Em seguida, foi armazenado por 24 horas em uma temperatura de $-20^{\circ} \mathrm{C}$ (Chaikouski et al., 2014).

O filmes biodegradáveis foram desenvolvidos no Laborátorio de Controle de Poluição da Universidade Paranaense (Umuarama, Paraná), através da técnica denominada de casting, onde é feita a dispersão, solubilização e gelatinização em um solvente com a formação de uma solução filmogênica (Yamashita et al., 2005). Portanto, foram desenvolvidos 4 filmes: A (4 gramas de amido contendo $30 \%$ de glicerol), AC ( 3 gramas de caseína +1 grama de amido contendo $30 \%$ de glicerol), AB (4 gramas de amido contendo $30 \%$ de glicerol $+1 \%$ de extrato de barbatimão) e ACB (3 gramas de caseína +1 grama de amido contendo $30 \%$ de glicerol $+1 \%$ de extrato de barbatimão). Para a preparação dos filmes biodegradáveis o amido foi gelatinizado em banho-maria a $85^{\circ} \mathrm{C}$ (Marconi-MA 039), e a caseína foi dissolvida em $25^{\circ} \mathrm{C}$.

A adição do glicerol e do extrato de barbatimão ocorrem após o resfriamento da solução até $30^{\circ} \mathrm{C}$. Todos os filmes biodegradáveis tiveram um volume final de $100 \mathrm{~mL}$, sendo retirado uma alíquota de $10 \mathrm{~mL}$ e colocada em placas de teflon, posteriormente estas soluções foram secas à $40^{\circ} \mathrm{C}$ em uma estufa.

A espessura foi determinada utilizando um micrômetro manual (Mitutoyo Série $193 \pm 0,001 \mathrm{~mm}$ ) (Bertan et al., 2005). 
Para a determinação da solubilidade em água, foi utilizada a metodologia descrita por Gontard et al. (1994), onde três discos de cada amostra de filme, com $2 \mathrm{~cm}$ de diâmetro foram cortados e submetidos à secagem por $24 \mathrm{~h}$ a $100{ }^{\circ} \mathrm{C}$ e pesados. Posteriormente, as amostras foram imersas em $50 \mathrm{~mL}$ de água destilada, onde foram mantidas por 24 h, sob agitação. Após, foi determinado o conteúdo da matéria seca não solubilizada através de filtração e secagem a $100^{\circ} \mathrm{C}$ por $24 \mathrm{~h}$. A solubilidade foi calculada como a relação entre a matéria seca final e a matéria seca inicial.

A opacidade dos filmes foi determinada pela metodologia de Park \& Zhao (2004), com a utilização de um espectrofotômetro (SHIMADZU UV-1650PC), onde os filmes foram cortados em retângulos ( 1 x 4 $\mathrm{cm}$ ) e aderidos a parede interna da cubeta do espectrofotômetro. Em seguida, foi varrida a faixa de comprimento de onda da luz visível, em 600 nm. A opacidade foi calculada através da absorbância dividida pela espessura da película.

Através de um colorímetro (CR 400 Konica Minolta), foram obtidos os valores de L*(luminosidade), a* (intensidade da cor verde a vermelho) e b*(intensidade da cor amarela a azul) e posteriormente foi determinada a variação total de cor $(\Delta \mathrm{E})$ (Pranoto, Salokhe \& Rakshit, 2005).

A microestrutura dos filmes foi analisada usando um microscópio eletrônico de varredura (MEV), (LEO 440, LEICA-ZEISS).

O delineamento foi inteiramente casualizado, com 5 repetições por tratamento. Os resultados foram submetidos a análise de variância (ANOVA), e ao teste $\mathrm{t}(\mathrm{LSD}) \mathrm{p}<0,05$. Todas as análises estatísticas foram realizadas utilizando o software BIOESTAT.

\section{RESULTADOS E DISCUSSÃO}

Os resultados da espessura, solubilidade em água e opacidade dos filmes de amido e caseína com a adição de extrato de barbatimão (Tabela 1).

A espessura dos filmes variou de 0,09 a 0,12 mm (Tabela 1), apresentando menor variação que Henrique, Cereda \& Sarmento (2008), que foi entre 0,06 e 0,12 $\mathrm{mm}$ em filmes de amido de mandioca.

A incorporação do extrato de barbatimão nos grupos A e AC, não apresentaram mudanças na espessura dos filmes $(\mathrm{P}>0,05)$. Os resultados foram similares a $\mathrm{Ma}$
\& Wang (2016) e Moradi et al. (2012) que não obtiveram mudanças na espessura de filmes biodegradáveis utilizando extratos de casca de uva. Segundo RojasGraü et al. (2007), a incorporação de óleos essenciais em filmes biodegradáveis também não modificaram a sua espessura.

A solubilidade em água é fundamental para uma potencial aplicação dos filmes, porém a solubilidade ideal depende da sua finalidade, uma alta solubilidade é desejada para filmes que serão consumidos simultaneamente com o alimento, enquanto que uma baixa solubilidade é desejada para aplicação de filmes como embalagens, em alimentos com uma umidade elevada (Rhim, Weller \& Rham, 1998). De acordo com os resultados apresentados na Tabela 1, a solubilidade em água decresceu com a adição do extrato de barbatimão nos filmes de amido e caseína, o grupo AB apresentou a menor solubilidade se comparado aos demais tratamentos $(20,41 \%)$, isso se deve ao constituinte majoritário do barbatimão, os taninos, que formam ligações hidrofóbicas com as proteínas e polissacarídeos (Rodrigues et al., 2013). Resultados similares foram obtidos por Ghelejlu, Esmaili \& Almasi (2016) para a solubilidade em água de filmes biodegradáveis com a adição de extratos vegetais. Sendo assim, os filmes com extrato de barbatimão possuem um maior potencial para serem usados como embalagens.

Os resultados mostram que os filmes com barbatimão apresentaram maior opacidade em relação aos demais, com 5,57 e 6,64 Abs600. $\mathrm{mm}^{-1}$, respectivamente, se diferenciando dos demais tratamentos sem a presença do extrato de barbatimão. Resultados similares já foram relatados em filmes com extrato de orégano e alecrim (Gómez-Estaca, et al., 2009) e extrato de chá verde (Siripatrawan \& Harte, 2010).

A coloração é considerada um importante fator na embalagem de alimentos e possui grande influência na aceitação dos consumidores (Ghelejlu et al., 2016). De acordo com os parâmetros apresentados na Tabela 2, a claridade dos filmes A foi afetado pela incorporação do extrato de barbatimão, enquanto que a incorporação não afetou a claridade dos filmes AC. Os filmes com a presença de caseína (AC e ACB) apresentaram os maiores valores de *L $(54,88$ e 55,22$)$, ou seja, filmes mais claros, o que pode ser explicado pela presença da caseína. 
Tabela 1 - Valores médios de espessura, solubilidade em água e opacidade em filmes compostos de amido e caseína com extrato de barbatimão

\begin{tabular}{lccc}
\hline Filmes & Espessura $(\mathrm{mm})$ & Solubilidade em água $(\%)$ & Opacidade $\left(\right.$ Abs600.mm $\left.{ }^{-1}\right)$ \\
\hline A & $0,09^{\mathrm{b}}$ & $35^{\mathrm{b}}$ & $1,63^{\mathrm{b}}$ \\
AC & $0,11^{\mathrm{a}}$ & $40^{\mathrm{a}}$ & $2,52^{\mathrm{b}}$ \\
AB & $0,09^{\mathrm{b}}$ & $20,41^{\mathrm{d}}$ & $5,57^{\mathrm{a}}$ \\
ACB & $0,12^{\mathrm{a}}$ & $25,5^{\mathrm{c}}$ & $6,64^{\mathrm{a}}$ \\
\hline
\end{tabular}

Médias com letras iguais na mesma coluna não diferem significativamente a 5\% de probabilidade pela análise de variância ANOVA, em um critério de teste $\mathrm{t}(\mathrm{LSD})$.

O extrato de barbatimão proporcionou um aumento na variação de verde a vermelho ( $\mathrm{a}^{*}$ ) apenas nos filmes de amido. Tendências similares na adição de extratos vegetais em filmes biodegradáveis já foi observada por Wang et al. (2016). Porém, para os filmes de amido e caseína, a incorporação do extrato de barbatimão não proporcionou diferenças significativas. Pires et al. (2011) e Atarés et al. (2011) concluíram que óleos essenciais não alteram o parâmetro $\mathrm{a}^{*} \mathrm{em}$ filmes biodegradáveis.

Todos os filmes apresentaram valores positivos para o parâmetro $b^{*}$, indicando uma coloração amarelada, justificada pela presença do amido nos filmes (Rocha et al., 2014). O extrato de barbatimão diminuiu o parâmetro $\mathrm{b}^{*}$ nos filmes de amido (A e AB), porém nos filmes de amido e caseína ( $\mathrm{AC}$ e $\mathrm{ACB}$ ), não ocorreram mudanças. Sendo que os grupos com a presença de caseína, apresentaram os maiores índices. Para Moradi et al. (2012) as mudanças na coloração são influenciadas pelo tipo dos compostos adicionados que se ligam estruturalmente as soluções formadoras dos filmes.

As micrografias dos filmes $\mathrm{A}, \mathrm{AC}, \mathrm{AB}$ e $\mathrm{ACB}$ estão apresentadas na Figura 1. Os filmes Ae AC demonstraram uma superfície lisa (sem rachaduras), porém apresentaram multicamadas, que são típicas de filmes de amido (López et al., 2011). O filme AC não apresentou grandes aglomerados sugerindo uma boa dispersão entre a caseína e o amido. Com a adição do extrato de barbatimão a superfície dos filmes se tornou mais áspera (Figura 1). Os mesmos padrões de superfície em filmes de caseína com outros extratos vegetais foram encontrados por Kadam et al. (2015) e pode ser atribuída aos complexos protéicos formados, devido a ligação entre compostos fenólicos e carboidratos (Rattaya, Benjakul \& Prodpran, 2009). A estrutura heterogênea dos filmes $\mathrm{AB}$ e $\mathrm{ACB}$ contribuiu para o aumento de sua opacidade, Carpiné et al. (2016) também observaram aumento na opacidade de filmes biodegradáveis ao incorporarem extrato de Yucca schidigera.
Tabela 2 - Valores médios da colorimetria de filmes de amido e caseína com extrato de barbatimão

\begin{tabular}{lcccc}
\hline Filmes & $\mathrm{L}^{*}$ & $\mathrm{a}^{*}$ & $\mathrm{~b}^{*}$ & $\Delta \mathrm{E}$ \\
\hline A & $46,29^{\mathrm{c}}$ & $-11,86^{\mathrm{b}}$ & $32,75^{\mathrm{b}}$ & $62,22^{\mathrm{b}}$ \\
AC & $54,88^{\mathrm{a}}$ & $-9,09^{\mathrm{a}}$ & $36,43^{\mathrm{ab}}$ & $66,71^{\mathrm{ab}}$ \\
AB & $51,65^{\mathrm{b}}$ & $-9,56^{\mathrm{a}}$ & $24,84^{\mathrm{c}}$ & $53,46^{\mathrm{c}}$ \\
ACB & $55,22^{\mathrm{a}}$ & $-9,07^{\mathrm{a}}$ & $37,49^{\mathrm{a}}$ & $67,49^{\mathrm{a}}$ \\
\hline
\end{tabular}

*Médias com letras iguais na mesma coluna não diferem significamente a 5\% de probabilidade pela análise de variância ANOVA, em um critério de teste t (LSD).

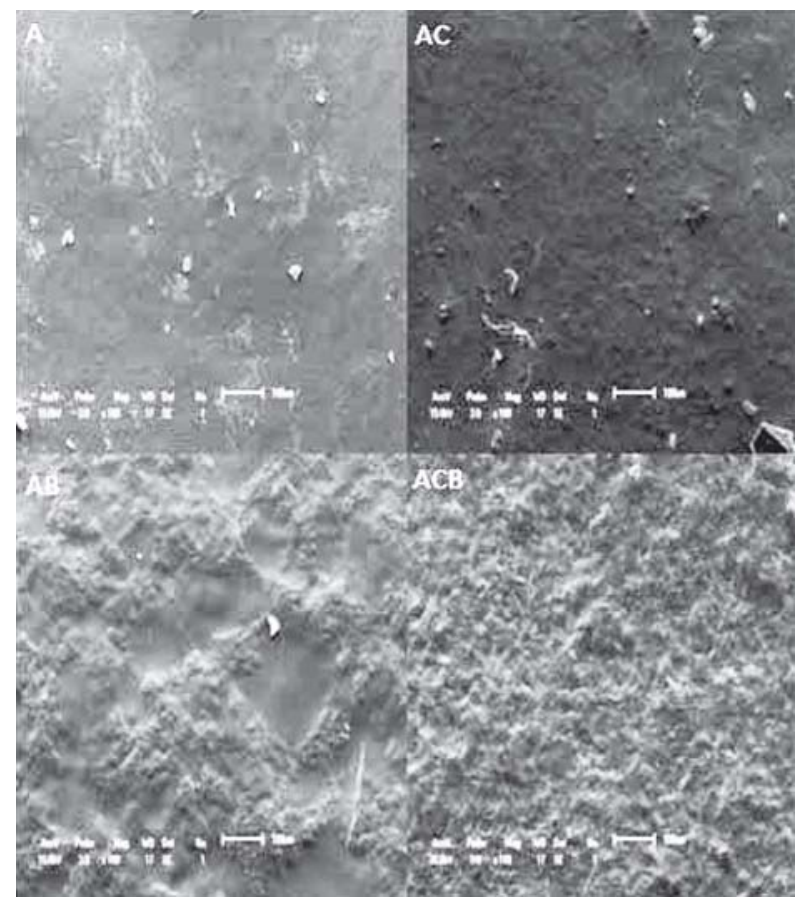

Figura 1 - Micrografias de mev de filmes de amido e caseína com extrato de barbatimão: (a) amido; (ac) amido e caseína; (ab) amido com extrato de barbatimão; (acb) amido e caseína com extrato de barbatimão. 


\section{CONCLUSÕES}

Os resultados demonstraram que a adição do extrato de barbatimão em filmes de amido e caseína não afetou a sua espessura, porém deixou os filmes menos solúveis e mais opacos.

A incorporação de extrato de barbatimão afeta a claridade de filmes de amido e caseína, deixandoos mais escuros e amarelados.

A morfologia dos filmes de amido e caseína é influenciada pela adição do barbatimão, deixando a superfície mais áspera.

\section{LITERATURA CITADA}

BERTAN, L. C.; TANADA-PALMU, P. S.; SIANI, A. C. et al. Effect of fatty acids and 'Brazilian elemi' on composite films based on gelatin. Food Hydrocolloids, v. 19, n. 1, p. 73-82, 2005.

CARPINÉ, D.; DAGOSTIN, J. J. A.; DE ANDRADE, E. F. et al. Effect of the natural surfactant Yucca schidigera extract on the properties of biodegradable emulsified films produced from soy protein isolate and coconut oil. Industrial Crops and Products, v. 83, p. 364-371, 2016.

CASTRO, A. H. F.; PAIVA, R.; DE ALVARENGA, A. A. et al. Calogênese e teores de fenóis e taninos totais em barbatimão [Stryphnodendron adstringens (mart.) coville]. Ciência e Agrotecnologia, v. 33, n. 2, 2009.

CHAICOUSKI, A.; SILVA, J. E.; TRINDADE, J. L. F. et al. Determinação da quantidade de compostos fenólicos totais presentes em extratos líquido e seco de erva-mate (Ilex paraguariensis). Revista Brasileira de Produtos Agroindustriais, v. 16, n. 1, p.33-41, 2014.

CORREA-BETANZO, J.; JACOB, J. K.; PEREZPEREZ, C. et al. Effect of a sodium caseinate edible coating on berry cactus fruit (Myrtillocactus geometrizans) phytochemicals. Food Research International, v. 44, n. 7, p. 1897-1904, 2011.

FERNANDES, A. P. S.; COSTA, J. B.; SOARES, D. $\mathrm{S}$. B et al. Aplicação de filmes biodegradáveis produzidos a partir de concentrado protéico de soro de leite irradiado. Pesquisa

Agropecuária Tropical, v. 45, n. 2, p. 192199, 2015.
GHELEJLU, S. B.; ESMAIILI, M.; ALMASI, H.

Characterization of chitosan-nanoclay bionanocomposite active films containing milk thistle extract. International Journal of Biological Macromolecules, v. 86, p. 613$621,2016$.

GÓMEZ-ESTACA, J.; MONTERO, P.; FERNANDÉZMARTIN, F. et al. Physical and chemical properties of tuna-skin and bovine-hide gelatin films with added aqueous oregano and rosemary extracts. Food Hydrocolloids, v. 23, p. 1334-1341, 2009.

GONTARD, N.; DUCHEZ, C.; CUQ, J. et al. Edible composite films of wheat glúten and lipids: Water vapor permeability and other physical properties. International Journal of Food Science Technology, v. 29, n. 1, p. 39-50, 1994.

HENRIQUE, C. M.; CEREDA, M. P.; SARMENTO, S. B. S. Características físicas de filmes biodegradáveis produzidos a partir de amidos modificados de mandioca. Ciência e Tecnologia de Alimentos, v. 28, n. 1, p. 231-240, 2008.

KADAM, S. U.; PANJAK, S. K.; TIWARI, B. K. et al. Development of biopolymer-based gelatinand casein films incorporating brown seaweed Ascophyllum nodosum extract. Food Packaging and Shelf Life, v. 6, p. 68-74, 2015.

LAFARGUE, D.; LOURDIN, D.; DOUBLIER, J.-L. Film-forming properties of a modified starch/kcarrageenan mixture in relation to its rheological behaviour. Carbohydrate Polymers, v. $70, \mathrm{n}$. 1, p. 101-111, 2007.

LÓPEZ, O. V.; LECOT, C. J.; ZARITZKY, N. E. et al. Biodegradable packages development from starch based heat sealable films. Journal of Food Engineering, v. 105, n. 2, p. 254-263, 2011.

MA, Q.; WANG, L. Preparation of a visual pHsensing film based on tara gum incorporating cellulose and extracts from grape skins. Sensors and Actuators B: Chemical, v. 235, p. 401407, 2016 .

MARTINS, J. T.; CERQUEIRA, M. A.; VICENTE, A. A. Influence of á-tocopherol on physicochemical properties of chitosan-based films. Food Hydrocolloids, v. 27, n. 1, p. 220-227, 2012. 
MORADI, M.; TAJIK, H.; ROHANI, S. M. R. et al. Characterization of antioxidant chitosan film incorporated with Zataria multiflora Boiss essential oil and grape seed extract. LWT - Food Science and Technology, v. 46, p. 477-484, 2012.

MOURE, A.; CRUZ, J. M.; FRANCO, D. et al. Natural antioxidants from residual sources. Food Chemistry, v. 72, p. 145-171, 2001.

DO NASCIMENTO, A. M.; GUEDES, P. T.; CASTILHO, R. O. et al. Stryphnodendron adstringens (Mart.) Coville (Fabaceae) proanthocyanidins quantitation by RP-HPLC. Brazilian Journal of Pharmaceutical Sciences, v. 49, p. 549-558, 2013.

DE OLIVEIRA, D. R.; FERREIRA JÚNIOR, W. S. F.; BITU, V. C. N. et al. Ethnopharmacological study of Stryphnodendron rotundifolium in two communities in the semi-arid region of northeastern Brazil.

Brazilian Journal of Pharmacognosy, v. 24, n. 2, p. 124-132, 2014.

PARK, S.; ZHAO, Y. Incorporation of mineral or vitamin into chitosan-based films. Journal of Agricultural and Food Chemistry, v. 52, n. 7, p. 1933-1939, 2004.

PIRES, C.; RAMOS, C.; TEIXEIRA, G. et al. Characterization of biodegradable films prepared with hake proteins and thyme oil. Journal of Food Engineering, v. 105, p. 422-428, 2011.

PRANOTO, Y.; SALOKHE, V. M.; RAKSHIT, S. K. Physical and antibacterial properties of alginatebased edible film incorporated with garlic oil. Food Research International, v.38, n. 3, p. 267-272, 2005.

RATTAYA, S.; BENJAKUL, S.; PRODPRAN, T. Properties of fish skin gelatin film incorporated with seaweed extract. Journal of Food

Engineering, v. 95, n.1, p. 151-157, 2009.

RHIM, J. W.; WELLER, C. L.; HAM, K. S. Characteristics of chitosan films as affected by type of solvent acid. Food Science and

Biotechnology, v. 7, n. 4, p. 263-268, 1998.
ROCHA, G. O.; FARIAS, M. G.; DE CARVALHO, C. W. P. et al. Filmes compostos biodegradáveis a base de amido de mandioca e proteína de soja. Polímeros, v. 24, n. 5, p. 587-595, 2014.

RODRIGUES, D. F.; MENDES, F. F.; NORONHA FILHO, A. D. F. et al. O extrato da casca de barbatimão, Stryphnodendron adstringens (Martius) Coville, na cicatrização de feridas em animais. Enciclopédia Biosfera, v. 9, p. 1583-1601, 2013.

RODRÍGUEZ, F. J.; GALOTTO, M. J.; GUARDA, A. et al. Modification of cellulose acetate films using nanofillers based on organoclays.

Journal of Food Engineering, v. 110, n. 2, p. 262-268.

ROJAS-GRAÜ, M. A.; AVENA-BUSTILLOS, R. J.; OLSEN, C. et al. Effects of plant essential oils and oil compounds on mechanical, barrier and antimicrobial properties of alginate-apple puree edible films. Journal of Food

Engineering, v. 81, p. 634-641, 2007.

SIRIPATRAWAN, U.; HARTE, B. R. Physical properties and antioxidant activity of na active film from chitosan incorporated with Green tea extract. Food Hydrocolloids, v. 24, p. 770$775,2010$.

VERMEIREN, L.; DEVLIEGHERE, F.; VAN BEEST, M. et al. Developments in the active packing of foods. Trends in Food Science and Technology, v. 10, p. 77-86, 1999.

WANG, H.; HU, D.; MA, Q. et al. Physical and antioxidant properties of flexible soy protein isolate films by incorporating chestnut (Castanea mollissima) bur extracts. LWT - Food Science and Technology, v. 71, p. 33-39, 2016.

YAN, Q.; HOU, H.; GUO, P. et al. Effects of extrusion and glycerol content on properties of oxidized and acetylated corn-starch-based films. Carbohydrate Polymers, v. 87, n. 1, p. 707 $712,2012$.

Recebido para publicação em 22/4/2018 e aprovado em 30/6/2018. 